\title{
Les risques naturels d'origine glaciaire : inventaire dans les Alpes françaises et typologie
}

\author{
Natural risks of glacial origin : \\ an inventory in the french Alps and typology
}

\author{
par Anne Buisson, Christophe Dumas, Louis Reynaud, François Valla, \\ Cemagref - Etna et L.G.G.E.-C.N.R.S., Grenoble
}

Several terrain investigations, with specialized organizations such as services R.T.M., the P.G.H.M., allow us to count nearly 100 events which have occurred in the French Alps during 200 last years. The whole of this inventory has been centralized and synthesized in the form of data sheets; each glacier having presented at least one event has a data sheet, containing a description of the glacier (localization and a photograph), and the description of the various listed events. The data sheets were gathered by massif (Mont Blanc, Vanoise, Ecrins and the South Alps massif). After having indexed the events of glacial origin, it appeared necessary to classify them; 3 principal categories thus are distinguished:

- drainings of glacial lakes,

- falls of ice (distinguishing the falls from seracs, repetitive, from the glacial tongues ruptures or the falls of suspended glaciers, much rarer),

- ruptures of water pockets.

\section{I INTRODUCTION}

L'objectif de ce court exposé est de présenter l'état d'avancement d'un inventaire des événements d'origine glaciaire dans les Alpes françaises. Il est actuellement en cours de réalisation, grâce à un Contrat de Plan Etat-Région Rhône-Alpes (1998-1999), passé avec le Laboratoire de Glaciologie et Géophysique de l'Environnement (L.G.G.E. du C.N.R.S.) et le Cemagref de Grenoble.

Le but est de créer une vaste base de données recensant les événements d'origine glaciaire sur les 2 derniers siècles. Pour cela, nous avons eu recourt à des enquêtes auprès des différents organismes susceptibles de posséder les informations (services R.T.M., P.G.H.M....), à la consultation d'archives, et bien sûr à des contacts avec les spécialistes de certaines zones des Alpes.

Il est cependant important de garder à l'esprit que ce premier recensement ne représente qu'une partie de tous les événements, et ce pour deux raisons :

- parmi tous les événements qui se sont produits et qui se produisent actuellement, seuls certains ont été observés puis archivés (une grande partie passe inaperçue, ou n'est pas d'ampleur suffisante pour être notée),

- les archives traitant de ce sujet n'ont pas toutes été retrouvées ou dépouillées.

Cet inventaire est donc destiné à être complété et mis à jour périodiquement.

\section{II — L'INVENTAIRE DÉJÀ RÉALISÉ DANS LES ALPES FRANÇAISES}

\subsection{Présentation générale}

Depuis mars 1998, près de 100 événements ont été recensés en France et organisés en fiches récapitulatives (environ 40 autres événements ont par ailleurs été répertoriés en Europe grâce à des recherches bibliographiques).

Ces événements ont été portés sur une carte globale permettant de localiser les zones concernées (cf. figure 2) :

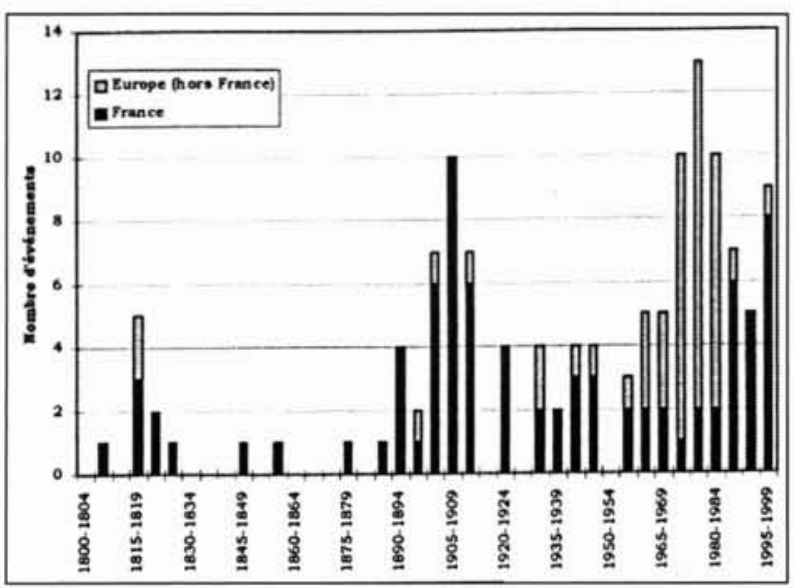

1. Distribution historique des événements recencés. 
pour chaque glacier " délictueux ", le (ou les) type(s) d’événements qui s'y sont produits ont été reportés (vidange de lac glaciaire, chute de glace, ou rupture de poche d'eau).

Il nous a ensuite paru intéressant de regrouper tous les événements survenus en France et en Europe sur un histogramme montrant leur répartition au cours du temps ( $\boldsymbol{c f}$. figure 1).

A première vue, le graphique met en évidence plusieurs périodes de forte occurrence (pics).

Mais attention, ces pics ne sont pas forcément significatifs et leur interprétation nécessite quelques remarques :

- la collecte d'informations actuellement en cours va accentuer le pic des 25 dernières années,

- l'échantillonnage dépend du système d'observation,

- les événements sont inévitablement filtrés, en fonction de l'importance de l'événement et de ses conséquences,

- il y a une perte évidente d'information historique.

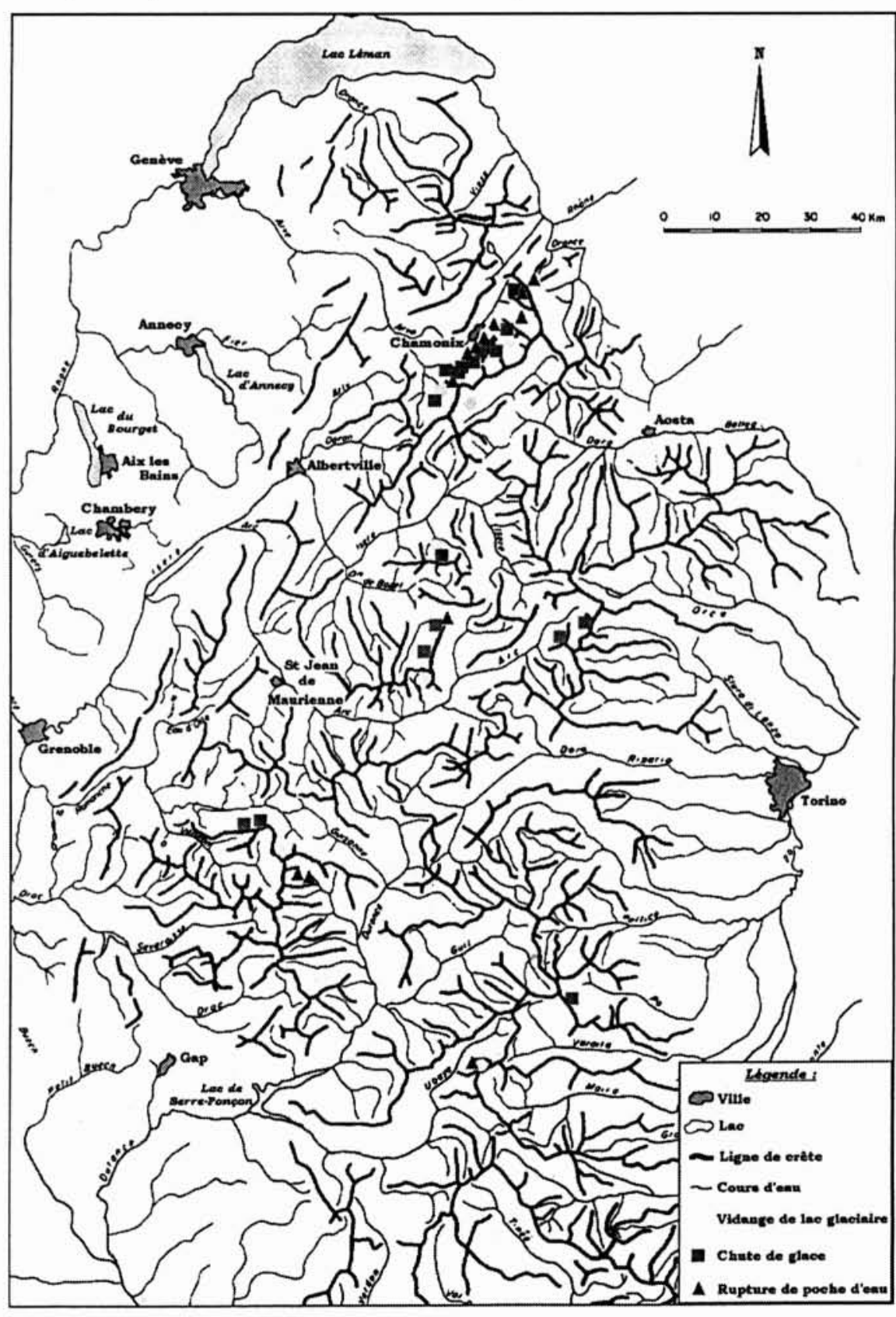

2. Carte de localisation des différents événements recensés.

\subsection{La fiche type}

Le principe retenu pour l'archivage des données est celui d'une fiche de renseignements par glacier, regroupant tous les événements qui s'y sont produits.

- Le recto de la fiche (voir figure 3) comporte principalement des indications concernant le glacier lui-même (nom du massif, localisation précise avec une carte, caractéristiques, photographie la plus récente possible...), ainsi qu'un (ou plusieurs) onglet(s) de couleur, indiquant le type d'événement recensé, et en bas de page, les dates des différents événements recensés.

- Le verso (voir figure 4) comporte le descriptif des différents événements recensés, séparant dans la mesure du possible le phénomène de ses conséquences. Lorsque les récits proviennent d'archives, un avis (en bleu) permet de se faire une idée de la qualité et de la fiabilité de l'information. Les sources d'information (témoignage, archives, ouvrage...) sont ensuite énumérées. Enfin, une dernière rubrique permet de préciser, s'il y a lieu, un contexte particulier, d'une évolution observée...

Lorsqu'un événement est suffisamment bien documenté, on peut envisager une page supplémentaire comportant plus de détails, et des photographies de l'événements.

\subsection{Le massif du Mont-Blanc}

Sur ce premier massif 70 événements (4 vidanges de lacs glaciaires, 36 chutes de glace, et 30 ruptures de poches d'eau) ont été recensés. La carte récapitulative (cf. figure 5) permet d'observer que de nombreux glaciers sont sujets à plusieurs types d'événements. Chaque type d'événement est représenté par une forme plus une couleur (vidange de lac glaciaire, chute de glace et rupture de poche d'eau).

Il est intéressant de noter la localisation des événements recensés : en majeure partie sur versant qui domine vallée ; en effet, ces événements ont été notés à cause de leur influence directe sur la vie de la vallée, mais il est certain que de nombreux autres se sont produits au cœur du massif qui n'ont pas été notés. Il y a donc une importante perte de données.

\subsection{Le massif de la Vanoise}

\section{L'inventaire est encore en cours.}

A l'heure actuelle, 10 événements ont été recensés précisément (2 vidanges de lacs glaciaires, 6 chutes de glace et 2 ruptures de poches d'eau), ainsi que de nombreuses chutes de séracs dont on connaît l'existence, mais pas les dates ni la fréquence d'occurrence (cf. figure 6 ). 


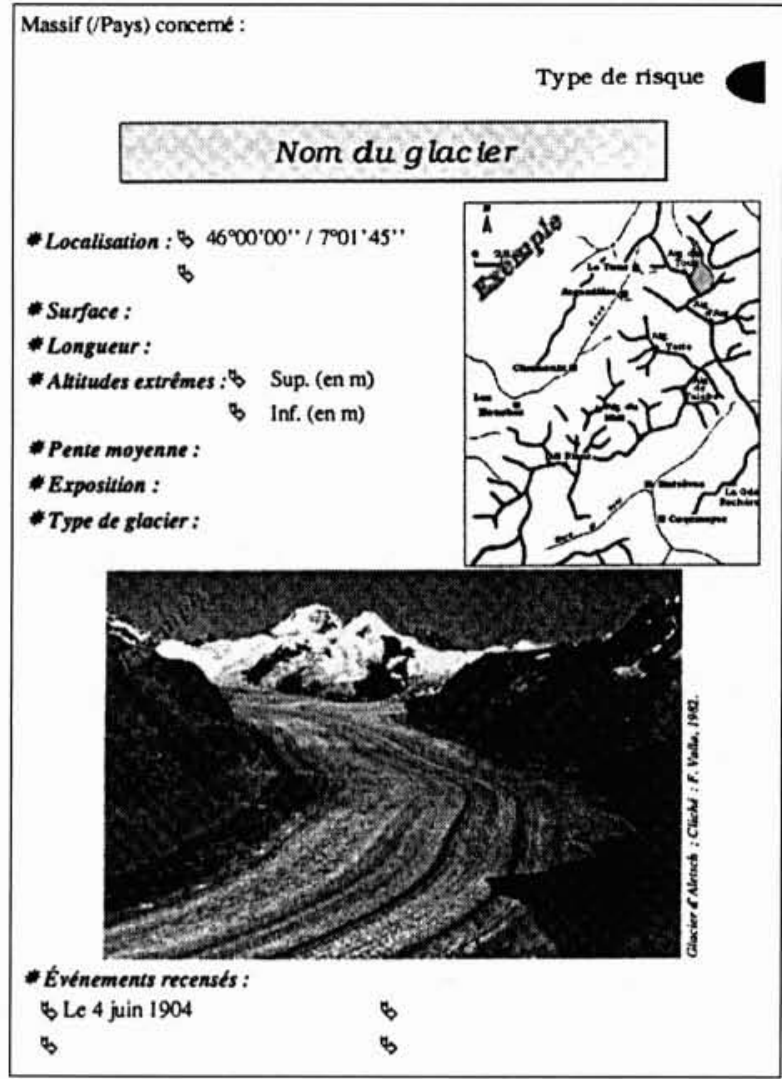

3. Recto d'une fiche de renseignements.

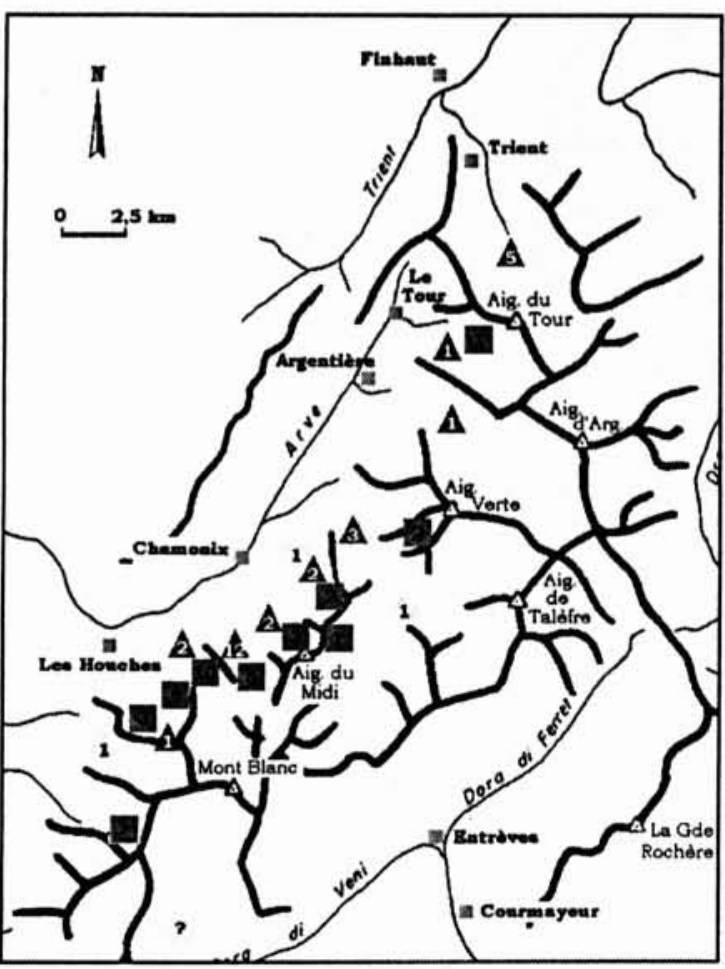

5. Détail des événements recensés (Mont-Blanc).
- Detail des foenements recensets :

(5) Date : le 4 juin 1904

$>$ Phenomène :un soudain afflux d'eau est sorti du glacier. L'hypothèse avancle a été celle de la rupture d'une poche d'eau située dans le glacier, car il n'y avait pas eu de précipitations au cours des jours précédents.

$>$ Consequences : mort de 2 personnes qui se trouvaient près du front.

L'information globale concernant cet événement est réduite; elle ne concerne que les consilquences et non l'événement en lui-même.

- Sonnces d'iuformation :

5 Vivian, R. 1975. Les glaciers des Alpes occidentales. These d'etat, Imprimerie Allier. Grenoble, p. 349.

- Commentaires :

Le glacier a été soupçonné d'une rupture de poche d'eau le 25 juillet 1996, mais cette hypothìse a été écarté en raison des précipitations abondantes qui l'ont précéde. In semble plus probable que la mise en charge soudaine de réseaux d'ecoulement intra-glaciaires habituels ait conduit a plusieurs petites debacles, attributes a tort a une rupture de poche d'eau.

4. Verso d'une fiche de renseignements.

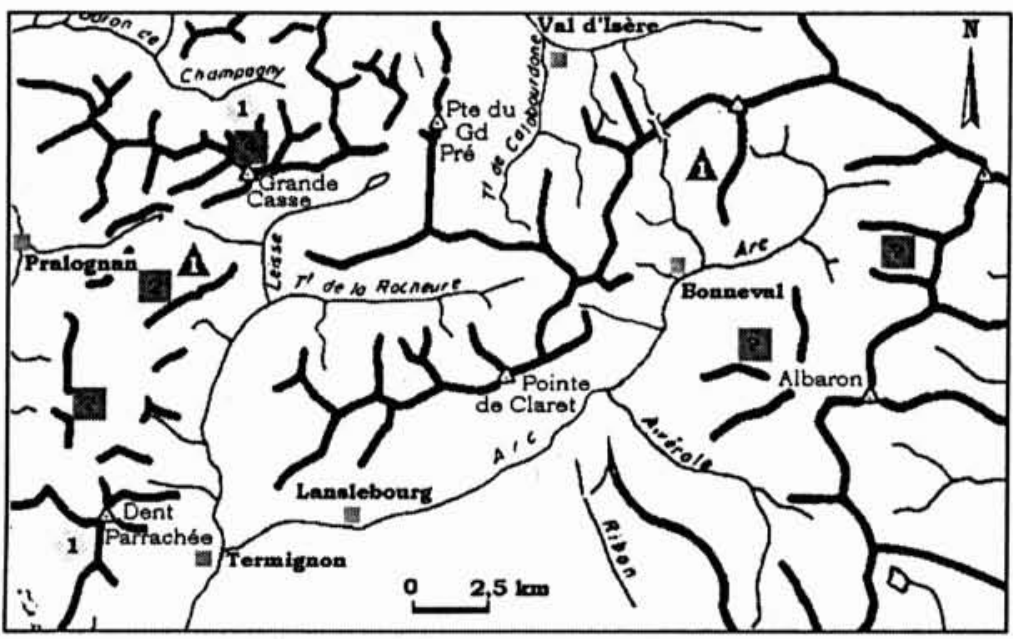

6. Détail des événements recensés (Vanoise)

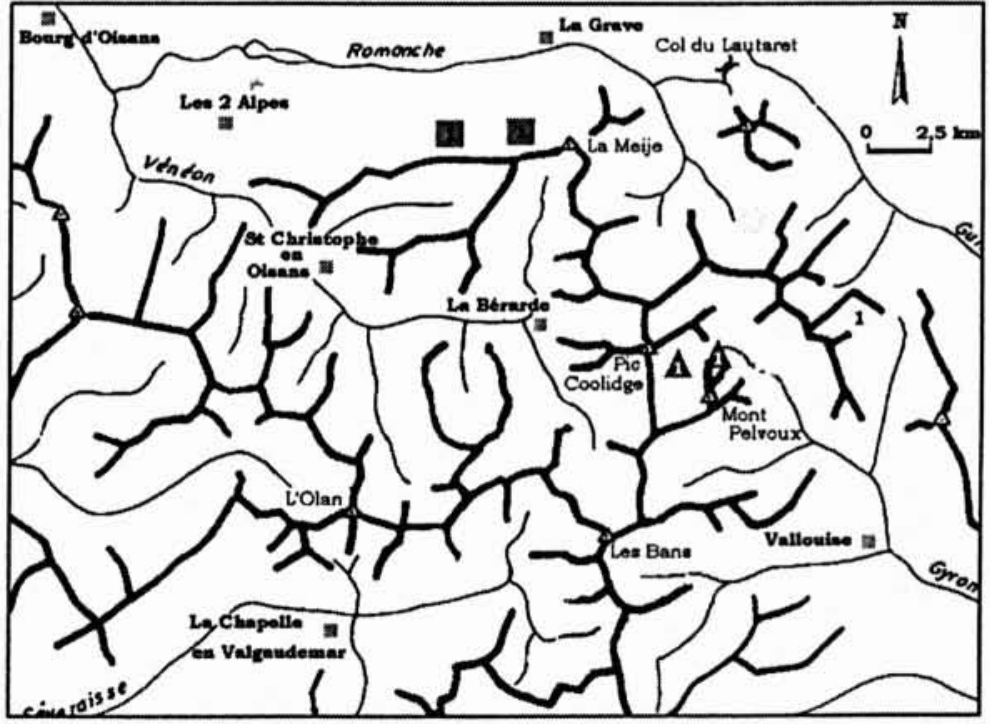

7. Détail des événements recensés (Ecrins). 


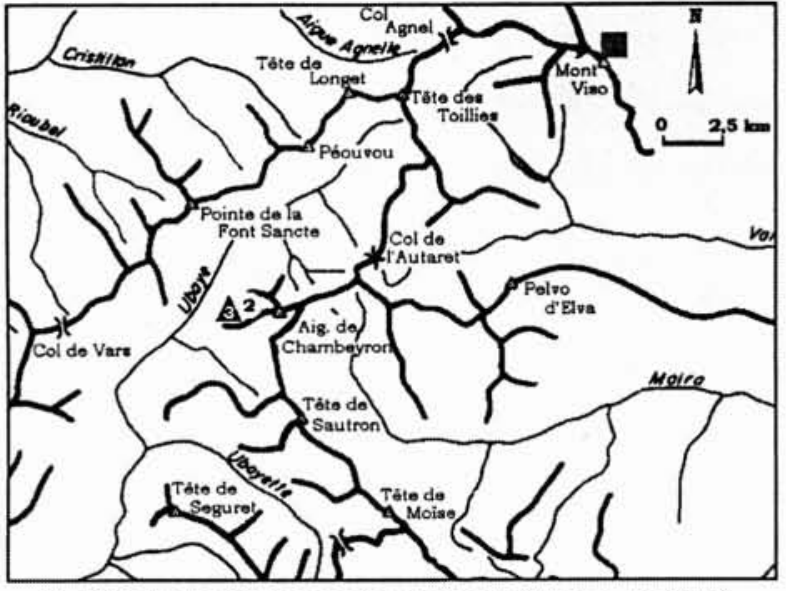

8. Détail des événements recensés (Alpes du Sud).

\subsection{Le massif des Ecrins}

Sur ce massif, 5 événements ont déjà été recensés (1 vidange de lac glaciaire, 2 chutes de glace et 2 ruptures de poches d'eau), plus le danger potentiel du lac du glacier d'Arsine (des travaux de sécurisation ont été réalisés en 1986 ; aujourd'hui un second lac se développe, et se déverse directement dans le canal d'évacuation construit en 1986) ; voir figure 7.

Comme en Vanoise, le recul prononcé des glaciers depuis plusieurs années est à l'origine de la création de lacs proglaciaires dont il faudra surveiller l'évolution.

\subsection{Les Alpes du Sud}

Bien qu'il ne reste que peu de glaciers dans cette région, 6 événements d'origine glaciaire ont été recensés (2 récentes vidanges de lacs glaciaires, la chute d'un glacier suspendu et 3 ruptures de poches d'eau), concernant deux glaciers seulement : le glacier du Coolidge (face nord du Viso) et celui du Fond de Chauvet (cf. figure 8).

\section{- 2.7 Les événements " enveloppe"}

Suite à l'inventaire des événements d'origine glaciaire en France, l'objectif est ici de montrer que ces phénomènes peuvent mettre en jeu des volumes énormes et être à l'origine de terribles catastrophes, comme en témoignent les 3 exemples qui suivent.

Il faut cependant distinguer les événements qui se produisent en France ou en Europe, dont l'ordre de grandeur est la centaine de victimes, des événements au niveau mondial, qui peuvent provoquer des milliers de victimes.

\subsubsection{La vidange du lac de Giétro (1818)}

Elle s'est produite le 16 juin 1818 à $16 \mathrm{~h}$. Des chutes de séracs répétées dans la vallée avaient donné naissance à un barrage important (1 $700000 \mathrm{~m}^{3}$ de glace, $\boldsymbol{c f}$. figure 9), retenant $27,5 \mathrm{Mm}^{3}$ d'eau. Au cours des travaux mis en œuvre pour vidanger artificiellement le lac, ce dernier s'est brutalement vidangé alors qu'il contenait encore 18 millions de $\mathrm{m}^{3}$. Cet énorme flot a dévasté la vallée, causant de nombreuses destructions matérielles, et plus de 50 victimes.

2.7.2 Les chutes de glace du Huascaran (1962 et 1970) :

- Le 10 janvier 1962: 3 millions de mètres cubes de

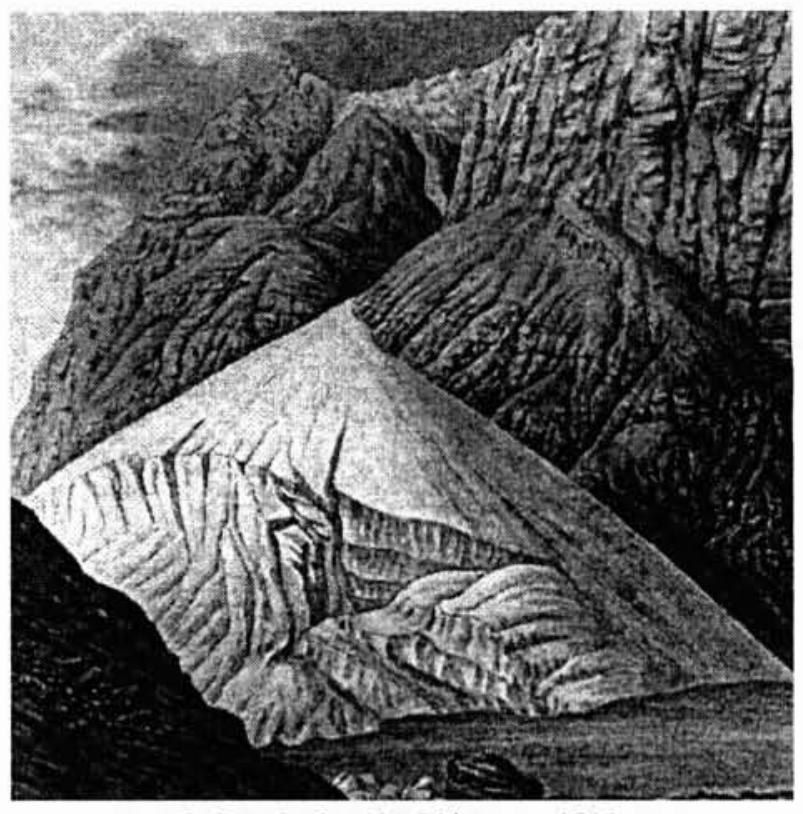

9. Le glacier de Giétro en 1818.

glace se sont détachés du sommet et ont dévalé le versant jusqu'à la petite ville de Ranrahirca. Environ 13 millions de $\mathrm{m}^{3}$ de mètres cubes de matériaux ont ainsi été transportés, tuant 4000 personnes.

- Le 31 mai 1970 : suite à un séisme, une importante chute de séracs et de rocher mélangés a donné naissance à une lave torrentielle qui a ravagé Yungay et Ranrahirca (50 millions de $\mathrm{m}^{3}$ de matériaux déposés), causant la mort d'au moins 15.000 personnes (cf. figure 10).

Nota : en Suisse, la chute du glacier Allalin (1 million de $\left.\mathrm{m}^{3}\right)$, a causé la mort de 88 personnes en détruisant le chantier de construction du barrage de Mattmark le 30 août 1965.

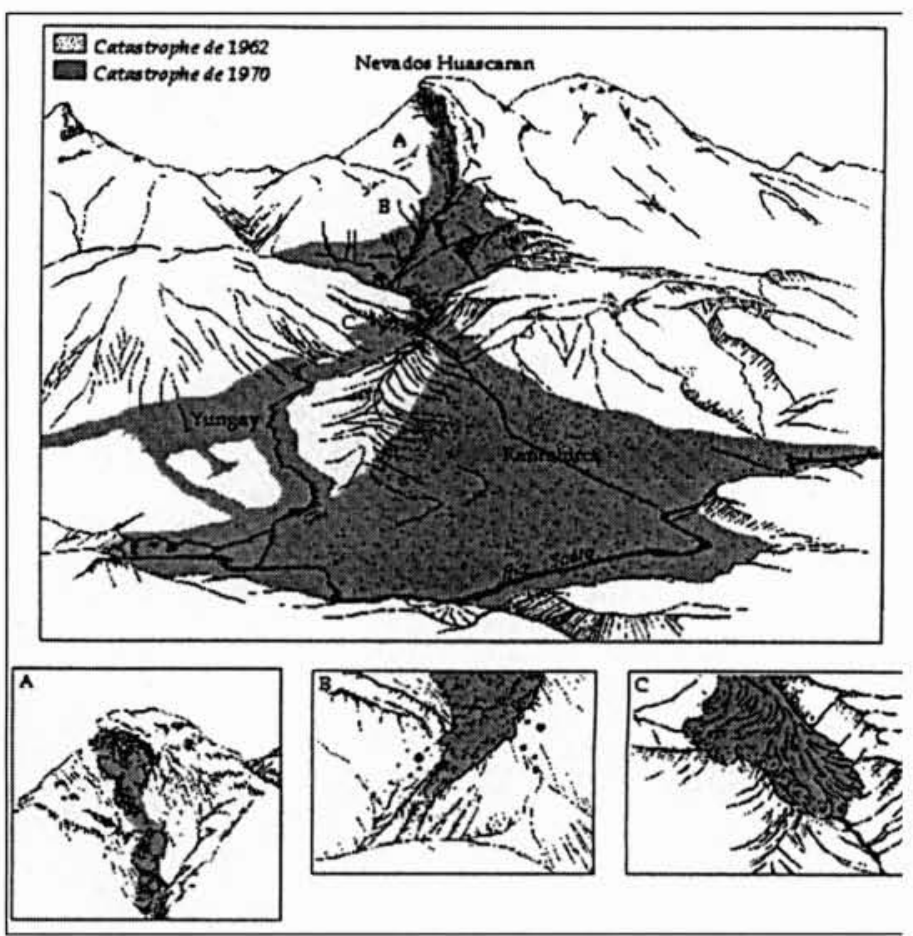

10. L'extension des dégâts (1962 et 1970). 


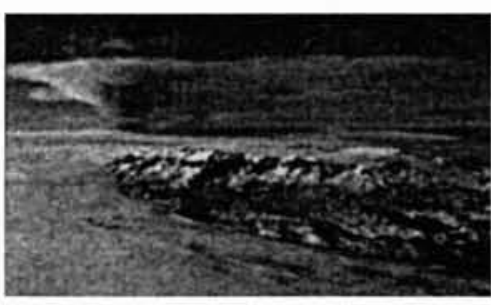

11. Le Vatnajökull

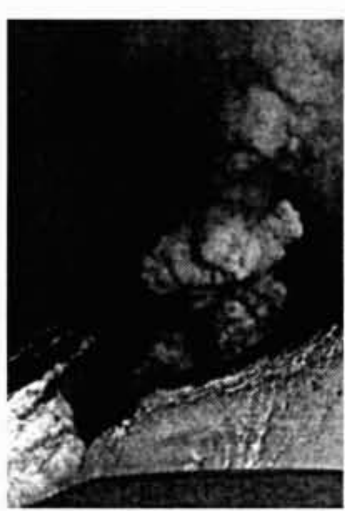

12. L'éruption volcanique

13. Le creux en surface après la vidange.

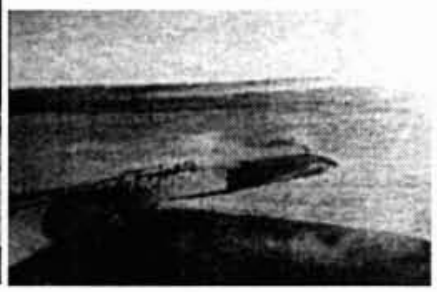

2.7.3 La rupture de poche d'eau du Vatnajökull (1996) :

Suite à une éruption volcanique sous glaciaire du Grimsvötn (figures 11, 12 et 13) qui s'est produite le 29 septembre, une gigantesque poche d'eau s'est formée, contenant près de 4 milliards de $\mathrm{m}^{3}$ d'eau.

La vidange de cette impressionnante poche s'est amorcée le 5 novembre 1996, et s'est poursuivie durant plusieurs jours. Le débit maximum a atteint $45000 \mathrm{~m}^{3} / \mathrm{s}$ pendant plusieurs heures. Au total, ce sont près de 100 millions de tonnes de matériaux qui ont été charriées.

Les conséquences n'ont été que de faible ampleur au regard de l'énormité des volumes mis en jeu ; en effet, l'événement était suivi et attendu, et des mesures préventives avaient été mises en place (le coût total des dégâts a tout de même atteint la somme de 31 millions de \$ 1996 !).

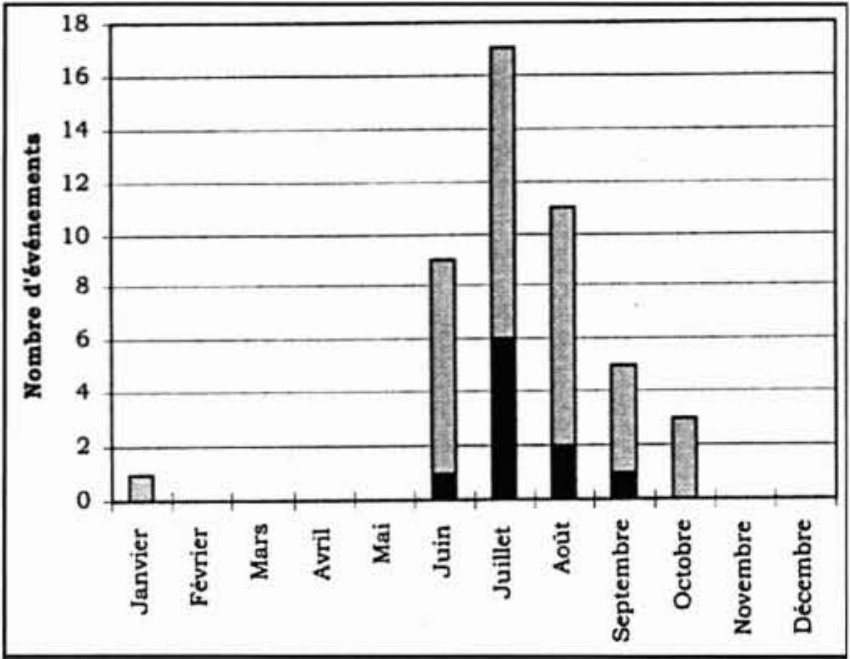

15. Distribution saisonnière des débâcles.

\section{III — UNE ÉBAUCHE DE TYPOLOGIE}

L'inventaire réalisé a mis en évidence différents types de phénomènes que l'on a tenté de classer en fonction de leur nature. Trois catégories principales se sont ainsi dégagées : les vidanges de lacs glaciaires, les chutes de glace et les ruptures de poches d'eau. Cette première approche de la typologie devrait permettre d'amorcer une réflexion sur les mécanismes relatifs à ces 3 catégories. Cependant, nous rappelons que les éléments présentés ici ne sont qu'une première approche de la typologie et que cette dernière demanderait du temps et des moyens spécifiques pour se développer.

\section{- 3.1 Les vidanges de lac glaciaire}

Il existe de nombreux modes de vidange d'un lac glaciaire ; l'exemple illustré ici (cf. figure 14) est celui d'un lac proglaciaire retenu par un barrage morainique, dont la vidange est causée soit par submersion, soit par un phénomène de renard.

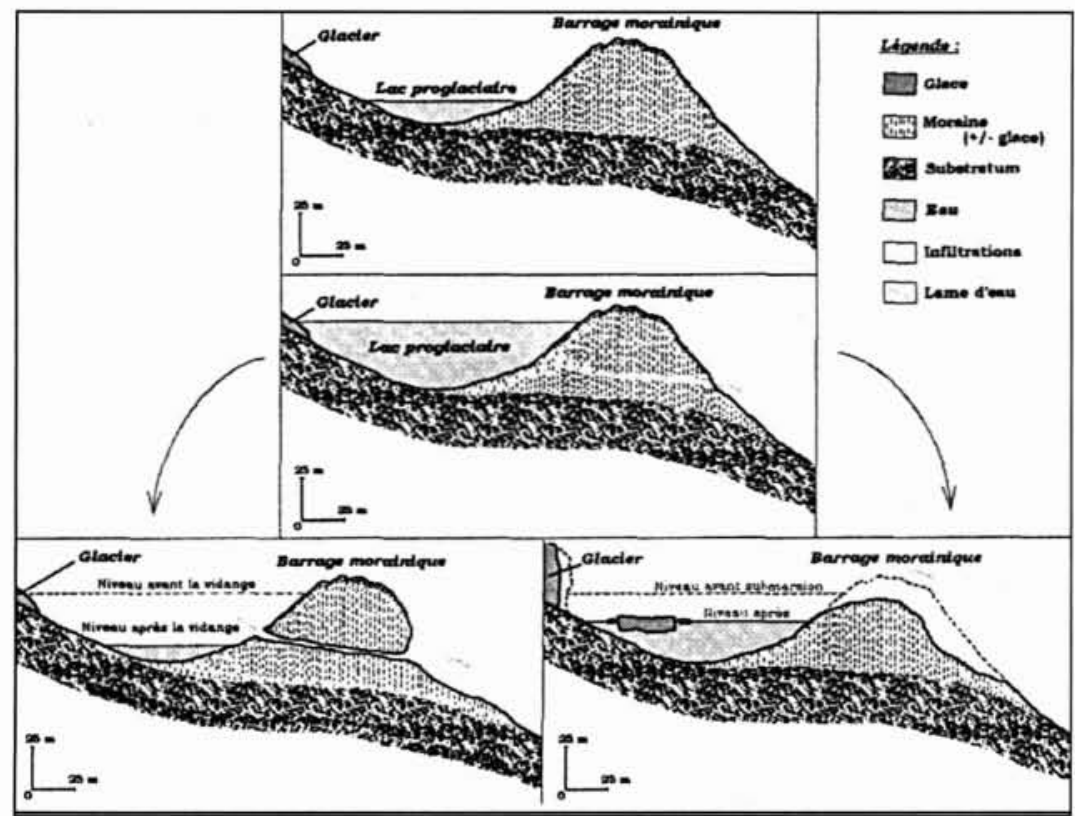

14. Schémas d'une vidange de lac glaciaire.
La vidange d'un lac est souvent soudaine et brutale. Les conséquences pour l'aval peuvent aller de l'inondation à une lave torrentielle dévastatrice.

Exemples français : glacier de Lépenaz (1818), glacier des Nantillons (1944), glacier de Lesché (1979)...

On remarque aussi que la distribution saisonnière des vidanges de lacs glaciaires (débâcles) n'est pas aléatoire (cf. figure 15) : il existe une période d'occurrence privilégiée durant la période estivale à laquelle on pouvait s'attendre dans la mesure où c'est celle où le niveau des lacs est maximal.

\subsection{Les chutes de glace}

Cette appellation générique comporte deux principaux sous-types :

- les chutes de séracs : phénomènes répétitifs qui peuvent se produire plusieurs 


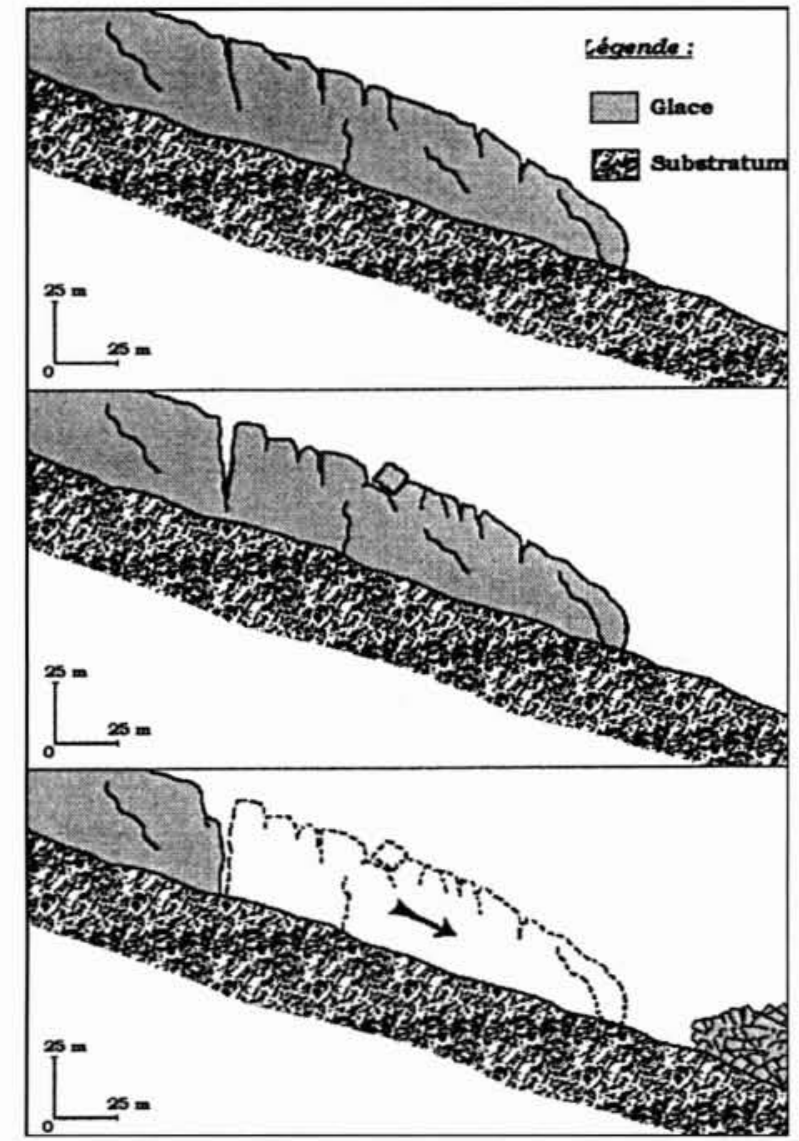

16. Schéma d'une rupture de langue glaciaire.

fois par an, dont le volume est limité à quelques milliers de mètres cubes de glace,

Exemples français : glacier de la Charpoua, glacier des Bossons...
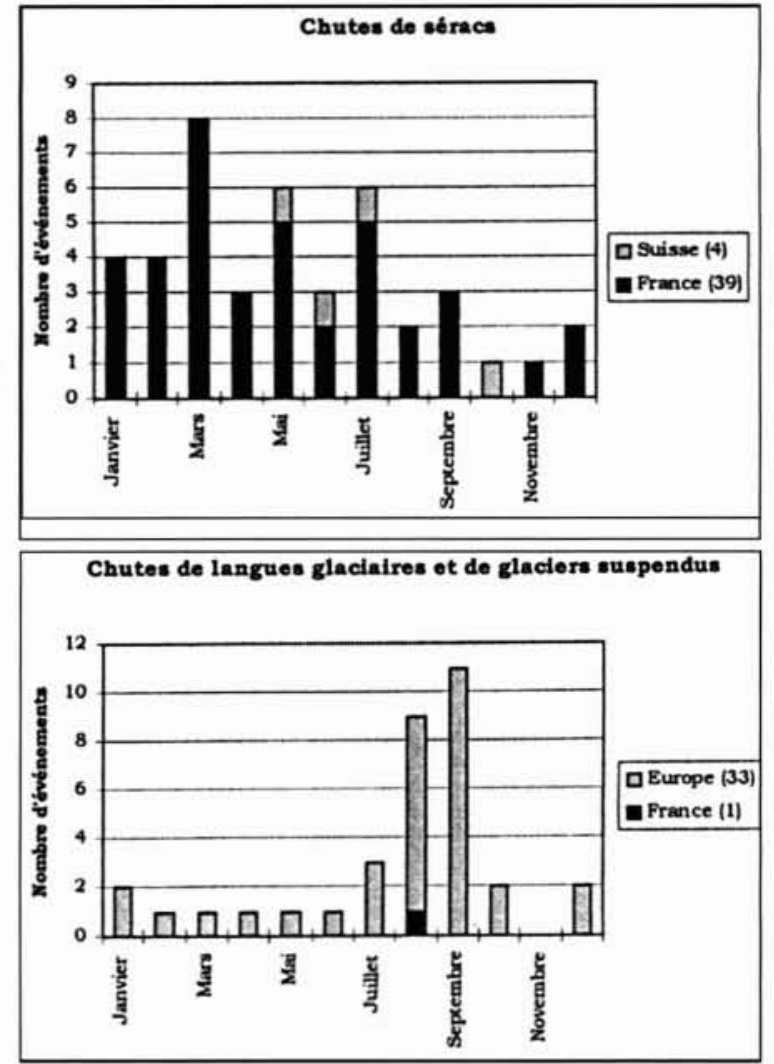

17. Distribution saisonnière des chutes de glace.

- les ruptures de langues glaciaires (cf. figure 16) et les chutes de glaciers suspendus : phénomènes exceptionnels, pouvant mettre en jeu des volumes de plusieurs millions de mètres cubes de glace.

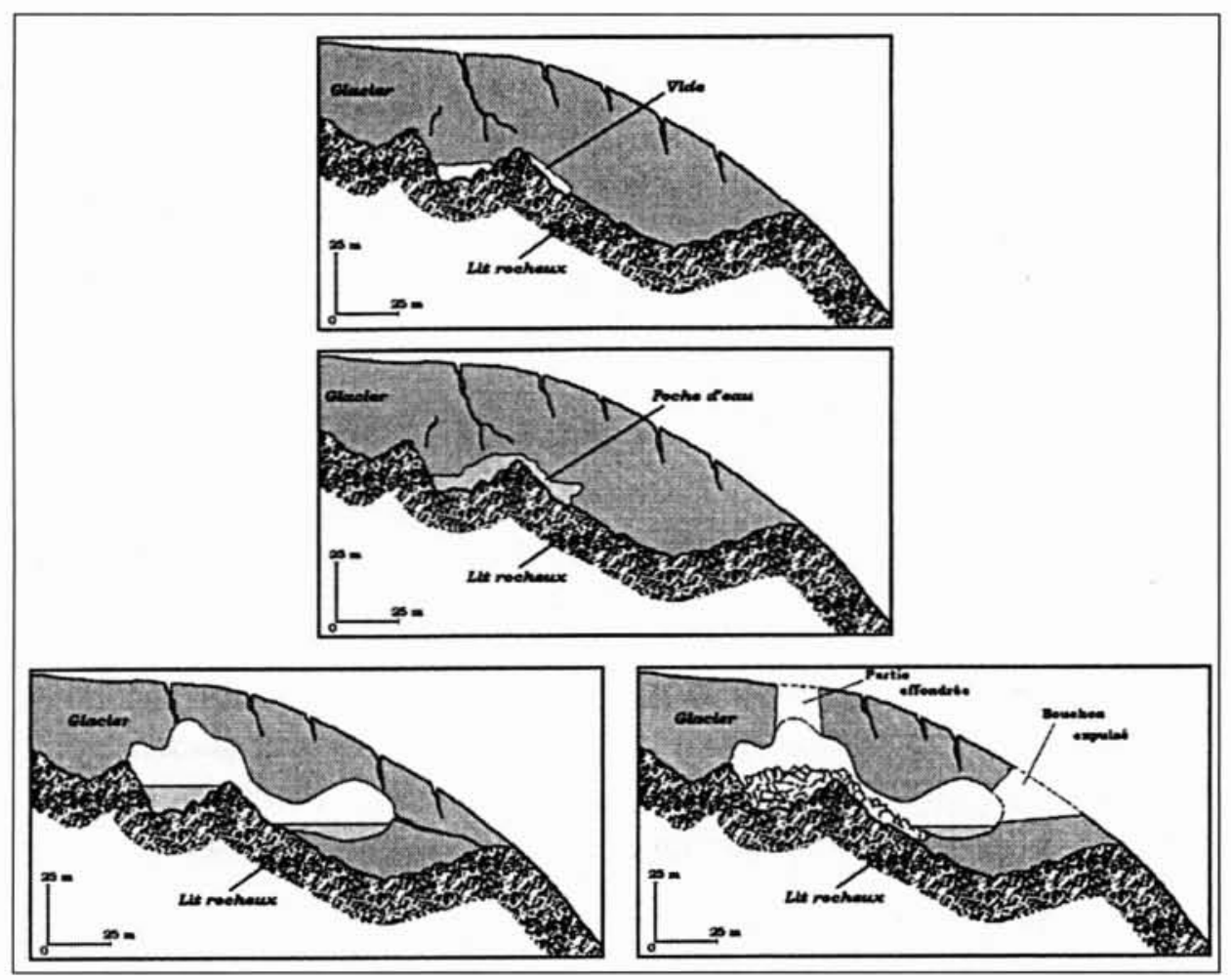

18. Schéma d'une rupture de poches d'eau. 


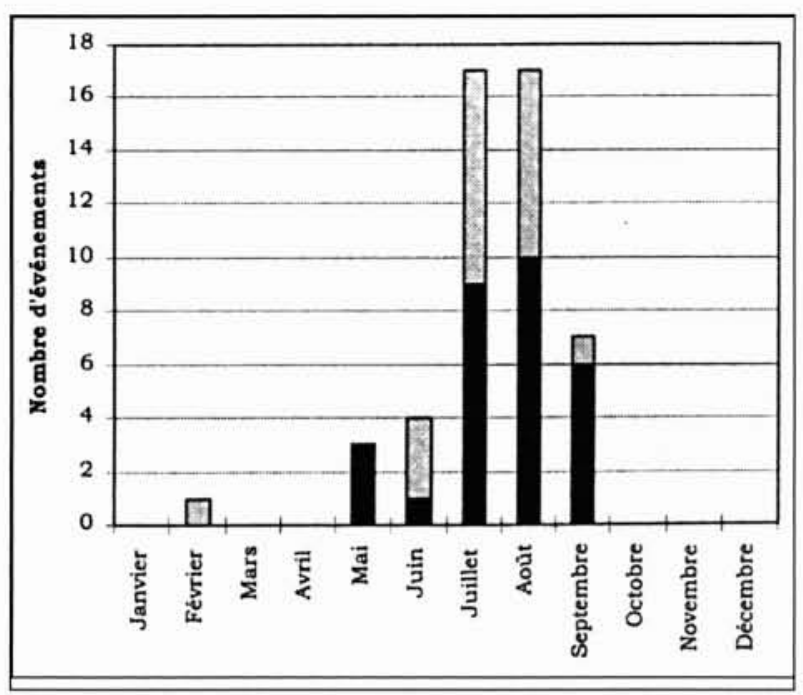

19. Distribution saisonnière des ruptures de poches d'eau.

Exemples : glacier du Tour (1949), glacier Allalin - Suisse (1965), glacier Coolidge (Mont Viso) - Italie (1989)...

Distribution saisonnière des chutes de glace (cf. figure 17) : - les chutes de séracs: elles se produisent toute l'année, et aucune période d'occurrence particulière n'a été mise en évidence.

- les ruptures de langues glaciaires et chutes de glaciers suspendus : il semble que la période estivale soit plus propice à ce genre de chute de glace, ce qui semble logique compte tenu de la lubrification basale du glacier par la fonte.

\subsection{Les ruptures de poche d'eau (cf. figure 18)}

C'est le plus mal connu des 3 types de risques. Le phénomène échappe à toute observation durant sa phase de développement ; il est d'autant plus dangereux qu'il est totalement imprévisible à l'heure actuelle.

On ne sait pas encore très bien pourquoi et comment les poches se forment, mais on suppose que les étapes sont les suivantes : création d'un vide, remplissage et agrandissement de la poche, puis rupture (souvent brutale).

Exemples français : glacier de Tête-Rousse (200 morts en 1892), glacier des Bossons (à plusieurs reprises), glacier Noir (1958), glacier de l'Ouille Noire (1989)...

On remarque également que la distribution saisonnière présente une période d'occurrence privilégiée en été (cf. figure 19), liée à la fonte nivale puis glaciaire.

\section{IV $\square$ CONCLUSION}

L'Inventaire en l'état actuel doit être complété et mis à jour périodiquement. L'intérêt d'une telle base de données est de rassembler de nombreuses informations dont l'analyse devrait permettre de mieux comprendre les mécanismes des différents types d'événements d'origine glaciaire, dans l'objectif d'une prévention future efficace.
Note : Le rapport inventaire concernant les événements glaciaires des Alpes françaises est consultable auprès du service documentaire du Cemagref de Grenoble.

\section{Bibliographie}

[1] ALEAN, J. C., 1985. Ice avalanche activity and mass balance of a highaltitude hanging glacier in the swiss Alps. Annals of Glaciology, 6, p. 248-249.

[2] ASSIER, A., 1990. Les glaciers du Viso. Société Hydotechnique de France, section glaciologie-nivologie. Réunion des 15 et 16 mars, Grenoble.

[3] ASSIER, A. ET M. EVIN, 1998. The july 25th 1997 outburst at the Chauvet glacier, Haute Ubaye, France. Société Hydrotechnique de France, section glaciologie-nivologie. 2d Alpine Glaciological Meeting Grenoble.

[4] BACHMANN, R.C., 1979 (édition française). Glaciers des Alpes. Payot, Lausanne, 320p.

[5] CHAPOUTOT, P., 1998, L'Epena, montagne secrète de Vanoise. Imprimerie l'Edelweiss, Bourg St Maurice, 61p.

[6] DUTTO, F., GODONE, F, ET MORTARA, G., 1991. L'écroulement du glacier supérieur de Coolidge (Paroi nord du Mont Viso, Alpes occidentales). Revue de Géographie Alpine, n², p. 7-18.

[7] GLAISTER, R.M., 1951. The ice slide on the glacier du Tour. Journal of Glaciology, 1, (9), p. 508-509.

8] HAEBERLI, W., 1983 : Frequency and characteristics of glacier floods in the swis alps. Annals of Glaciology, 4, p. 85-90.

[9] HAEBERLI, W, ALEAN, J.C., MULLER, P. et FUNK, M., 1989. Assessing risks from glacier hazards in high mountain regions : some experiences in the Swiss Alps. Annals of Glaciology, 13, p. 96-102.

[10] HAEFELI, R., 1966. Note sur la classification, le mécanisme et le contrôle des avalanches de glace et des crues glaciaires extraordinaires. Association internationale d'hydrologie scientifique, 69, p. 316-325.

[11] LLIBOUTRY, L. 1965. Traité de Glaciologie. Tome II. Masson, Paris, $622 \mathrm{p}$.

[12] LLIBOUTRY, L., 1971. Les catastrophes glaciaires. La Recherche, 2 , (12), p. $417-425$.

[13] MARNEZY, A., 1981. Vidange brutale d'un lac proglaciaire en Vanoise. Revue de Géographie Alpine, LXIX, p. 489-494.

[14] MORALES, B., 1966. The Huascarãn avalanche in the Santa Valley, Peru. Association internationale d'hydrologie scientifique, 69, p. 304 315 .

[15] MOUGIN, P., 1925. Avalanches, débâcles et érosions glaciaires. Etudes glaciologiques, tome V, Imprimerie nationale. Paris, p. 159-177.

[16] Musée de Bagnes, 1988. 16 juin 1818, débâcle du Giétro. Pillet, Martigny, 200p.

[17] REYNAUD, L., 1982. La Mer de Glace et les glaciers du Mont-Blanc. Publication du comité scientifique des Réserves Naturelles du département de Haute-Savoie \& L.G.G.E. (C.N.R.S.). Imprimé par l'atelier Esope, Les Praz de Chamonix.

[18] REYNAUD, L., 1993. Le glacier Blane, le glacier Noir. BT nature. pemf. 39p.

[19] TUFNELL, L., 1984, Glacier Hazards. London and New York. Longman, $97 p$.

[20] VALLON, M., 1989. Evolution, water balance, potential hazards, and control of a pro glacial lake in the french alps. Annals of Glaciology, 13, p. 273-278.

[21] VIVIAN, R., 1975. Les glaciers des Alpes occidentales. Thèse d'état, Imprimeric Allier, Grenoble, 513p. 